\title{
Coupled analysis of material flow and die deflection in direct aluminum extrusion
}

\author{
W. Assaad* and H.J.M.Geijselaers ${ }^{\dagger}$ \\ ${ }^{*}$ Materials innovation institute, The Netherlands \\ w.assaad@m2i.nl \\ ${ }^{\dagger}$ Faculty of Engineering Technology, University of Twente, The Netherlands \\ H.J.M.Geijselaers@ctw.utwente.nl
}

\begin{abstract}
The design of extrusion dies depends on the experience of the designer. After the die has been manufactured, it is tested during an extrusion trial and machined several times until it works properly. The die is designed by a trial and error method which is an expensive process in terms of time and the amount of scrap. In order to decrease the time and the amount of scrap, research is going on to replace the trial pressing with finite element simulations. The goal of these simulations is to predict the material flow through the die. In these simulations, it is required to calculate the material flow and the tool deformation simultaneously. Solving the system of equations concerning the material flow and the tool deformation becomes more difficult with increasing the complexity of the die. For example the total number of degrees of freedom can reach a value of 500,000 for a flat die. Therefore, actions must be taken to solve the material flow and the tool deformation simultaneously and faster. This paper describes the calculation of a flat die deformation used in the production of a U-shape profile with a coupled method. In this calculation an Arbitrary Lagrangian Eulerian and Updated Lagrangian formulation are applied for the aluminum and the tool finite element models respectively. In addition, for decreasing the total number of degrees of freedom, the stiffness matrix of the tool is condensed to the contact nodes between the aluminum and the tool finite element models. Finally, the numerical results are compared with experiment results in terms of extrusion force and the angular deflection of the tongue.
\end{abstract}

Keywords: aluminum extrusion, die deflection, coupled analysis, static condensation PACS: $46.15 .-\mathrm{X}$

\section{INTRODUCTION}

Currently, the material flow and the tool deformation in an aluminum extrusion process are mostly calculated by a decoupled method $[1,4,3]$. In the decoupled method, the material flow of the aluminum is computed by a Eulerian formulation under the assumption of a rigid die. As soon as the simulation reaches the steady state, the reaction forces at the interface between the billet and the tool are exported and an Updated Lagrangian analysis is performed for the tool. In this method, the elasticity of the tool is not considered during the calculation of the material flow. Therefore, the coupled method is more desirable where the aluminum and the tool are calculated simultaneously. But this method requires more computer memory and more analysis time due to the large number of degrees of freedom. In this paper two different procedures for the coupled method are studied in the extrusion of a U- shape profile. The procedures include a full scale model, and a statically condensed tool. An Arbitrary Lagrangian Eulerian formulation will be applied in the coupled method for avoiding element distortion and following 


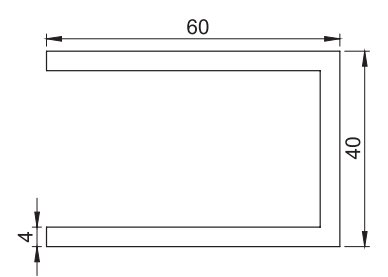

FIGURE 1. Profile (Dimensions in $\mathrm{mm}$ )

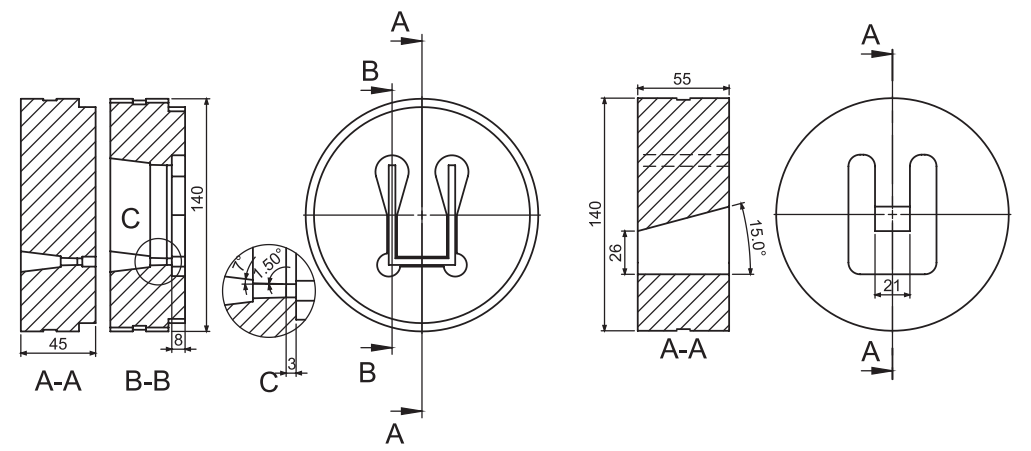

FIGURE 2. Die (left), Backer (right) (Dimensions in mm)

the moving boundaries. New mesh management options are implemented for the nodes at the interface between the aluminum and the tool finite element models. Finally, the numerical results are compared to the experimental results in terms of extrusion force and the angular deflection of the tongue. The computational time for the two procedures is checked. The angular deflection of the tongue for the die employed in extruding the profile is measured experimentally in an industrial environment [4].

\section{EXTRUSION OF A U-SHAPE PROFILE}

The U-shape profile shown in figure 1 is extruded from AA6060 billets by a 500 tons press with an extrusion ratio of 11.658. The die and the backer employed in the extrusion are shown in figure 2. A bolster with $100 \mathrm{~mm}$ length, $100 \mathrm{~mm}$ internal diameter and $199.5 \mathrm{~mm}$ outer diameter is supporting the die and backer.

\section{FULL SCALE MODEL}

\section{Boundary conditions and finite element model}

A stick boundary condition is applied on the billet's surface mating the inner container surface. The bolster's back surface is suppressed in the extrusion direction. At the interfaces between the backer and the bolster, the degrees of freedom are connected in the extrusion direction. The contact surface between the die and the backer is divided into two regions $C_{1}$ and $C_{2}$ where the degrees of freedom of the nodes belonging to 

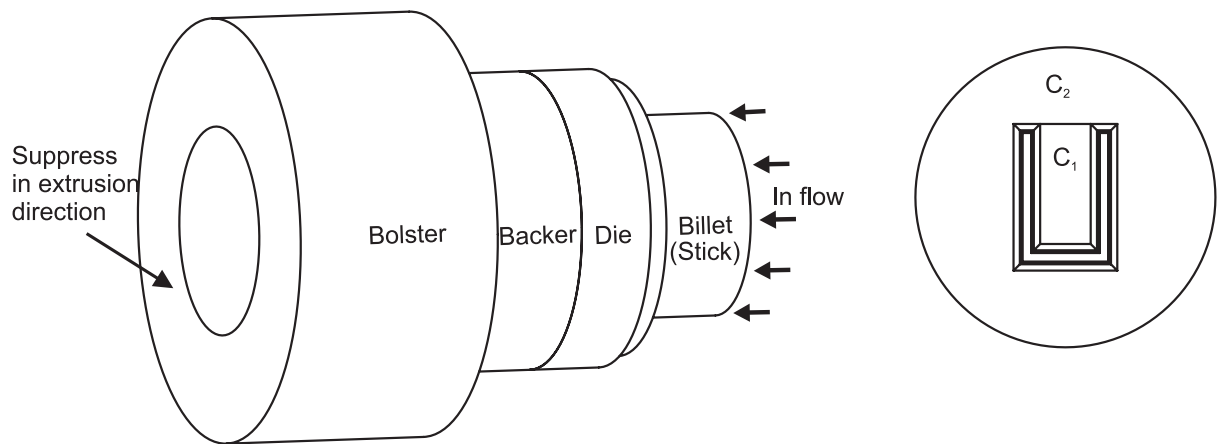

FIGURE 3. Boundary conditions applied on the full scale model (left), regions at the back side of the die (right)

TABLE 1. Constants used in Sellars-Tegart law for AA6060

\begin{tabular}{rccccc}
\hline $\mathbf{N}$ & $\mathbf{Q}[\mathrm{J} / \mathrm{mol}]$ & $\mathbf{A}\left[\mathrm{sec}^{-1}\right]$ & $\mathbf{R}[\mathrm{J} / \mathrm{K} . \mathrm{mol}]$ & $\boldsymbol{\alpha}\left[\mathrm{MPa}^{-1}\right]$ & $\mathbf{T}\left[\mathrm{K}^{\circ}\right]$ \\
\hline 4.22 & 187900 & $7.38 \mathrm{E} 11$ & 8.314 & 0.052 & 793 \\
\hline
\end{tabular}

region $C_{1}$ are connected in the extrusion direction only while those belonging to region $\mathrm{C}_{2}$ are connected in all directions. A conditional normal is defined at the bearing corner to prevent the change in material flow [2]. The degrees of freedom of the nodes at the interface between the die and billet are connected in all directions except at the bearing and bearing corner. They are connected in the plane normal to the extrusion direction at the bearing and in the normal direction at the bearing corner.

The whole model is discretized with 10 node tetrahedron elements at once in order to get node to node contact between the parts. Each node has translational degrees of freedom.

The behavior of aluminum alloy (AA6060) is described by von Mises viscoplastic material model. The relation between the flow stress and the equivalent viscoplastic strain rate is described by Sellars-Tegart law:

$$
\bar{\sigma}=\frac{1}{\alpha} \sinh ^{-1}\left(\left(\frac{\dot{\varepsilon}}{A} \exp \left(\frac{Q}{R T}\right)\right)^{\frac{1}{n}}\right)
$$

The constants are listed in table 1. The tool material (AISIH-13 steel) is described by elasto-plastic material model in which Voce hardening is used to describe the plastic behavior. The material properties at temperatures of $460 \mathrm{C}^{\circ}$ are listed in table 2 . 
TABLE 2. Material properties of the tool steel

\begin{tabular}{ccc}
\hline Young's Modulus $\left[\mathrm{N} / \mathrm{mm}^{2}\right]$ & Yield Stress $\left[\mathrm{N} / \mathrm{mm}^{2}\right]$ & Poisson's Ratio \\
\hline $1.75 \mathrm{E} 05$ & 850 & 0.29 \\
\hline
\end{tabular}

\section{Mesh management options}

In the ALE method the displacement of the mesh is decoupled from the material displacements. Different mesh displacement specifications are applied to the boundary and inner nodes of the billet volume in order to prevent element distortion due to large deformation.
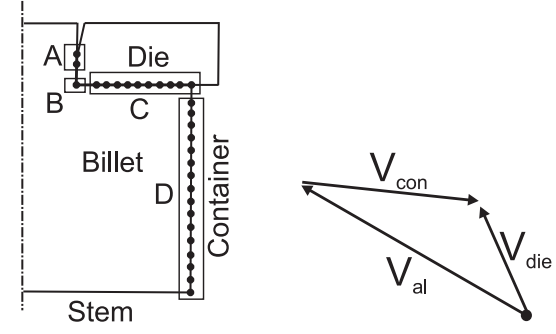

FIGURE 4. The boundaries where the mesh displacement options are applied (left), Convective displacements of nodes in sets A and B (right)

Figure 4 displays the three different boundaries of the billet with the extrusion tool. The nodes belonging to sets $\mathrm{A}$ and $\mathrm{B}$ are relocated to the location of their corresponding nodes on the die as shown in figure 4 . The nodes belonging to set $\mathrm{C}$ are made to follow the deformation of the die. The nodes belonging to set $\mathrm{D}$ do not move since the material is assumed to stick to the container wall.

The centering method discussed in [5] is applied for the internal nodes in the aluminum volume. Here a distinction is made between element corner nodes and element mid-side nodes.
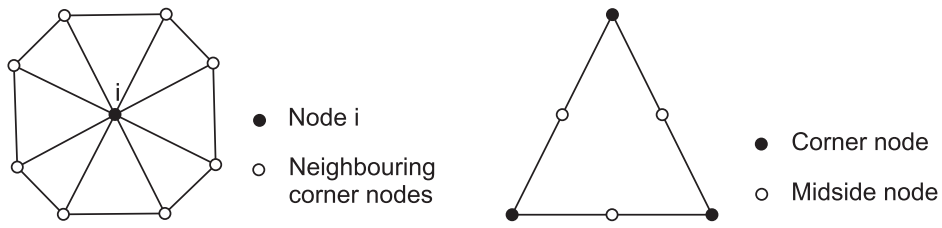

FIGURE 5. An inner node with its neighboring corner nodes (left), Corner and mid-side nodes (right)

The mesh displacement of the nodes in the interior of the mesh is evaluated by averaging the mesh displacements of the neighboring nodes.

$$
\overline{V_{g}^{i}}=\frac{1}{m} \sum_{j=1}^{m} V_{g}^{j}
$$




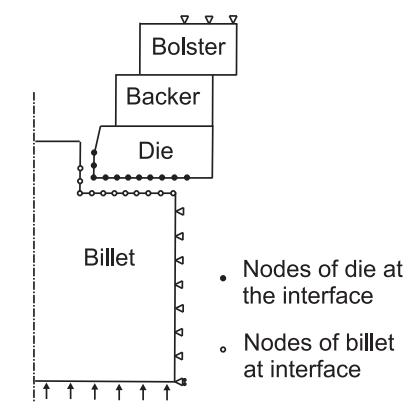

FIGURE 6. The tool nodes are condensed to nodes belonging to die-billet contact zone

Note that it is the mesh displacement which is averaged, not the mesh location. Initial mesh refinement is preserved.

The mesh displacement of the mid-side node is calculated from the average of the corresponding corner nodes (i-1) and (i+1) of the same element.

$$
\overline{V_{g}^{i}}=\frac{1}{2}\left(V_{g}^{i-1}+V_{g}^{i+1}\right)
$$

\section{STATICALLY CONDENSED TOOL}

The stiffness matrix of the tool which consists of die, backer and bolster is built separately. The displacement and force vectors of the tool's nodes are partitioned into two parts. First part described with subscript "o" corresponds to the nodes at the interface between the aluminum and the tool finite element models as shown in figure 6. Second part described with subscript " $i$ " corresponds to the internal and remaining boundary nodes of the tool.

Partitioning of the tool stiffness matrix and its condensation is described in the following equations:

$$
\begin{gathered}
{\left[\begin{array}{cc}
K_{o o} & K_{o i} \\
K_{i o} & K_{i i}
\end{array}\right] \cdot\left[\begin{array}{c}
u_{o} \\
u_{i}
\end{array}\right]=\left[\begin{array}{c}
F_{o} \\
F_{i}
\end{array}\right]=\left[\begin{array}{c}
F_{o} \\
0
\end{array}\right]} \\
\left(K_{o o}-K_{o i} K_{i i}^{-1} K_{i o}\right) u_{o}=\bar{K} u_{o}=F_{o}
\end{gathered}
$$

Since the original stiffness matrix of the tool is symmetric, only the matrices $K_{o o}, K_{i i}$ and $K_{i o}$ will stored. They are stored in sparse format. The condensed stiffness matrix $\bar{K}$ is calculated by a sparse solver for example Mumps or Sun performance instead of inverting the matrix $K_{i i}$.

Finally the upper half of $\bar{K}$ and the corresponding list of nodes numbers are imported into the FE program. The condensed stiffness matrix $\bar{K}$ is added to the global stiffness matrix as an element stiffness matrix. This element with a large number of degrees of freedom is designated by superlement. The boundary conditions at the interface between the aluminum and the condensed tool are applied. During the simulation the right hand 
TABLE 3. Comparison between full scale model, statically condensed tool and experimental results

\begin{tabular}{lccc}
\hline & Full scale model & Statically condensed tool & Experiment \\
\hline Extrusion force [MN] & 1.16 & 1.15 & 1.2 \\
Velocity [mm/sec] & 61.73 & 61.85 & 61.78 \\
Tongue deflection [mrad] & 7.2 & 7.2 & 7 \\
Degrees of freedom & 124371 & 69300 & \\
Non zero numbers & 4581982 & 86268436 & \\
Calculation time [hr] & 6.5 & 69 & \\
\hline
\end{tabular}

side of the superelement is updated by multiplying the total displacement of its nodes with its stiffness matrix.

\section{RESULTS AND CONCLUSION}

Table 3 shows the results of the procedures of the coupled method in comparison with the experimental results. The full scale model and the statically condensed tool have good agreement with the experimental results. Although the statically condensed tool reduces the number of degrees of freedom, it consumes more cpu time than the full scale model due to an increase in the amount of non zero numbers. The full scale model is more efficient to a certain number of degrees of freedom.

\section{ACKNOWLEDGMENTS}

This research was carried out under project number MC4.05221A in the framework of the Material innovation institute research program in the Netherlands.

\section{REFERENCES}

1. W. Assaad, and H.J.M.Geijselaers, 3-D Numerical Simulation of Direct Aluminum Extrusion and Die Deformation, Proceeding of Extrusion Technology 2008.

2. W. Assaad, and H.J.M.Geijselaers,Boundary Conditions Applied on bearing corner in Direct Aluminum Extrusion, International Journal of Material Forming, Volume 2, Supplement 1, Springer Paris,pp. 77-80 .

3. B. Reggiani, L.Donati, and L. Tomesani, Evaluation of Different FE Simulation Codes in the Stress Analysis of Extrusion Dies, Proceeding Esaform 2010, Brescia, Italy .

4. W. Assaad, and H.J.M.Geijselaers, Measuring the deformation of a flat die by applying a laser beam on a reflecting surface, Advances on Hot Extrusion and Simulation of Light Alloys, Trans Tech Publications, Switzerland, 2009, pp. 197-204.

5. H. C. Stoker, Dissertation, Developments of the Arbitray Lagrangian-Eulerian Method in non Linear Solid Mechanics, Enschede, Netherlands, 1999. 